\title{
viewpoint
}

\section{Complexity in biology}

\author{
Exceeding the limits of reductionism and determinism using complexity theory
}

\section{Fulvio Mazzocchi}

$\mathrm{T}$ he ultimate aim of scientific research is to understand the natural world. In order to achieve this goal, Western science has relied on different cognitive strategies, including simplification, both in terms of analysis and explanation. As the British natural philosopher Sir Isaac Newton (1643-1727) put it, "Truth is ever to be found in the simplicity, and not in the multiplicity and confusion of things." In a way, examples of simplification include using idealized models, such as a 'perfect sphere rolling down a smooth plane in a vacuum'; conducting experiments in a strictly controlled environment such as the laboratory; analysing complex systems by reducing them to their individual parts; and generally by using a linear and deterministic concept of how the world, including life, works.

The French philosopher and mathematician René Descartes (1596-1650) was the first to introduce reductionism to Western thinking and philosophy. According to his view, the world can be regarded as a clockwork mechanism; to understand it, one need only investigate the parts and then reassemble each component to recreate the whole. Descartes' work was expanded by Newton (1643-1727) and ultimately culminated in the Principia Mathematica in 1687-one of the most influential science books ever written-in which Newton further advanced the idea of a 'clockwork universe'.

Since the time of Newton, classical mechanics has been regarded as the foundation of scientific research. Scientists, including biologists, have adopted the Newtonian approach both at the ontological level-in terms of their conception of the world and the things of which it is made-and the epistemological level-in terms of their approach to understanding those things. The Newtonian epistemology, in fact, states that scientific knowledge has to provide an objective representation of the external world. The world's apparent complexity can be resolved by analysis and reducing phenomena to their simplest components. "Once you have done that, [the evolution of phenomena] will turn out to be perfectly regular, reversible and predictable, while the knowledge you gained will merely be a reflection of that pre-existing order" (Heylighen et al, 2007).

Ever since Newton formulated the first laws of gravity, the conceptual model of the physical world had successfully described the shape, movements and actions of the objects within it. But as physicists began to explore especially the atomic and subatomic realms in the early twentieth century, their observations became partially meaningless. The new discoveries required a paradigm shift and a new intellectual framework to understand events at the subatomic level, which eventually resulted in quantum physics.

\section{Since the time of Newton, classical mechanics has been regarded as the foundation of scientific research}

As many of the molecular biologists in the 1950s came from physics, it is not surprising that they extended its classical approach to the study of living organisms. Molecular biology, with some exceptions (Westerhoff \& Palsson, 2004), has largely adopted a reductionistic view to explain biological systems according to the physical and chemical properties of their individual components. As Francis Crick (1916-2004) put it, "The ultimate aim of the modern movement in biology is to explain all biology in terms of physics and chemistry" (Crick, 1966). In due course, reductionism proved to be an extremely powerful analytical methodology and it enabled scientists to analyse many basic molecular and cellular processes.

\section{Complex systems exist at different levels of organization that range from the subatomic realm to individual organisms to whole populations and beyond}

Nonetheless, biologists might be reaching the limits of this approach. Despite their best efforts, scientists are far from winning the war on cancer, owing largely to the complex nature of both the disease and the human organism. The human brain is a complex, nonlinear system that defies all reductionistic and deterministic attempts to understand it (Singer, 2007). On a macro level, ecosystems and human societies present the same challenge. What is needed is a new approach to study these systems. Complexity theory can provide new conceptual tools that will inevitably question many of the assumptions of Newtonian science.

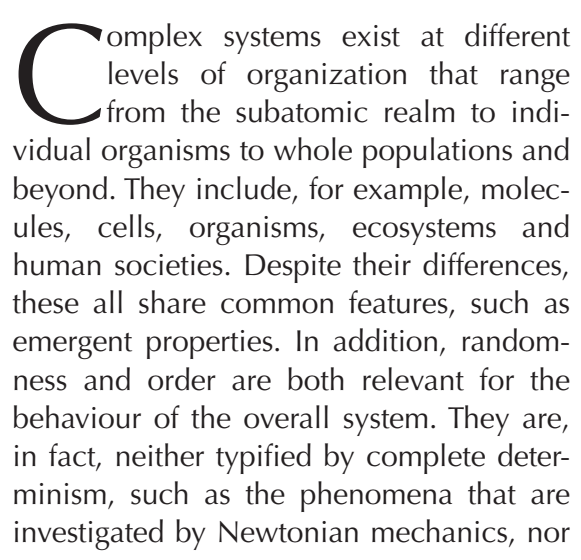




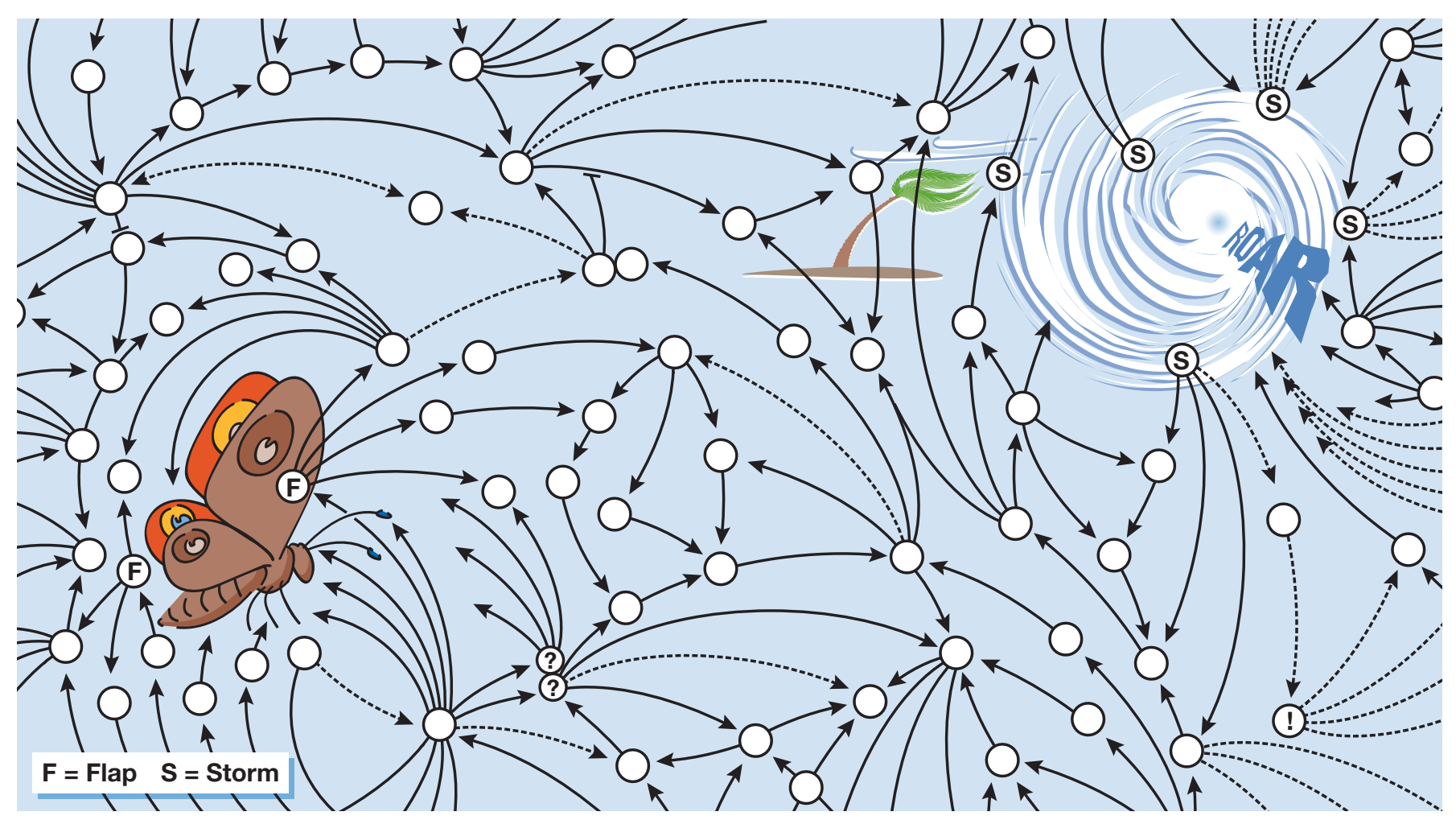

by total randomness, such as the subjects of statistical mechanics (Heylighen et al, 2007). Complex systems exist on the 'edge of chaos'. They might show regular and predictable behaviour, but they can undergo sudden massive and stochastic changes in response to what seem like minor modifications. The metaphor of the 'butterfly effect' whereby a single butterfly beating its wings can cause a storm-describes, for example, the dependence of a complex system on its initial conditions.

It is therefore important to analyse how the application of complexity theory can affect the study of biological systems, in the realm of molecular biology, too. This discussion is mainly concerned with the implications that these notions have for reductionism and determinism. Reductionism in particular can be distinguished into three types: ontological reductionism, which assumes that everything that exists in nature is constituted by a small set of primitive elements that behave in a regular and predictable manner; epistemological reductionism, which argues that fundamental concepts, laws and theories of a given level of organization can be derived from concepts, laws and theories pertaining to a lower level; and methodological reductionism, which claims that complex phenomena or systems can and should be understood by means of analysing their individual parts.

A $\mathrm{n}$ increasing number of scientists argue that the reductionist approach can no longer cope with both the enormous amount of information that comes from the so-called '-omics' sciences and technologies-genomics, proteomics, metabolomics and so on-and the astonishing complexity that they reveal. The assumption that complex biological systems can be completely explained by Descartes' clockwork model has been repeatedly questioned. As the Belgian biologist Marc Van Regenmortel commented, a move away "from the reductionistic viewpoint and toolset is a high priority for both biological and biomedical research" (Van Regenmortel, 2004).

First, the reductionist approach is not able to analyse and properly account for the emergent properties that characterize complex systems. The Greek philosopher Aristotle (384-322 вс) had already described emergence in his treatise Metaphysics as, "The whole is more than the sum of its parts." Thus, the whole system can neither be reduced nor deduced from the qualities of its individual parts; a view now held in a variety of scientific fields ranging from physics to sociology.

Biological organisms show emergent properties that arise from interactions both among their components and with external factors. For example, the properties of a protein are not equivalent to the sum of the properties of each amino acid. Proteins are able to specifically catalyse a chemical reaction, recognize an antigen or move along another protein polymer not only because their amino acids are arranged in a specific order, but also because their threedimensional structure and function are additionally determined by external factors.

The notion of emergence is also associated with the idea that the natural world consists of hierarchical levels of organization that range from subatomic particles to molecules, ecosystems and beyond (De Haan, 2006). Each level is both characterized and governed by emergent laws that do not appear at the lower levels of organization. This implies that, in order to explain the features and behaviour of a whole system, we require a theory that operates at the corresponding hierarchical level. For instance, emergent phenomena that occur at the level of the organism cannot be fully explained 
by theories that describe events at the level of cells or macromolecules. However, there are also various general features and characteristics - described in this article-that permeate all levels of organization and that allow the study of biological systems in the framework of complexity science.

\section{...the reductionist approach is not able to analyse and properly account for the emergent properties that characterize complex systems}

This hierarchical organization creates 'downward causation' (De Haan, 2006). While the behaviour of the whole is, to some degree, constrained by the properties of its components - 'upward causation' - the behaviours of its components are also constrained to a certain extent by the properties of the system. The behaviour of a cell, for example, is controlled both by the properties of its macromolecules and by the properties of the organ of which it is a part. The whole is not only more than the sum of its parts, but also less than the sum of its parts because some properties of the parts can be inhibited by the organization of the whole. From an epistemological point of view, this means that it is not enough to analyse each individual part (reductionism), nor is it enough to analyse the system as a whole (holism). A new model of scientific investigation to understand complex systems would require shifting the perspective from the whole to the parts and back again (Morin, 1990).

$\mathrm{S}$ econd, reductionism favours the removal of an object of study from its normal context. Experimental results obtained under given particular conditions or from a particular model—such as a mouse, in vitro cell cultures or computer models-are often extrapolated to more complex situations and higher organisms such as humans. But this extrapolation is at best debatable and at worst misleading or even hazardous. The failure of many promising drug candidates in clinical research shows that it is not always possible to transfer results from mice or even primates to humans (Horrobin, 2003).

More generally, the strategy of putting the object of study in an artificial, controlled and simplified experimental situation, even if it is done for heuristic purposes, is not neutral and has its price-it severs the essential link between the object and the natural context to which it belongs. Phenomena and properties that occur only in the living environment become obscured and skew the results. Field observations of chimpanzees, for example, have shown that these animals have certain complex behaviours that cannot be observed in an artificial setting but only in their natural environment (Goodall, 1986).

$\mathrm{T}$ hird, reductionism is also closely associated with determinism-the concept that every phenomenon in nature is completely determined by preexisting causes, occurs because of necessity, and that each particular cause produces a unique effect and vice versa. This, naturally, also sustains the idea of predictability. "When you are able to predict the development of a system from some predefined condition, then you have established a deterministic relation between the elements which constitute the system" (Emmeche et al, 1997). Nonetheless, complex biological systems cannot be fully understood on a purely deterministic basis. In fact, they often show nonlinear behaviour (De Haan, 2006).

As pointed out above, the individual components in complex systems interact in manifold ways, including highly dynamic regulatory and feedback mechanisms (Van Regenmortel, 2004). Within this framework, a single cause can produce multiple and unpredictable effects and even small fluctuations can have unexpected consequences. Linear casual explanations-that conceive reality as a linear succession of elementary events from cause to effect-are therefore possibly unable to describe how complex systems behave (Kellenberger, 2004).

Nevertheless, many molecular biologists still favour simple models with explanations based on a few individual factors. For example, knockout experimentswhich are widely used to determine the functional role of a gene-overemphasize the role of a single gene and are of limited use for understanding complex genetic networks. As a matter of fact, many knockout experiments have no or unexpected effects because any given gene product is only a part of a complex network.

In addition, other phenomena can cause these unforeseen findings such as gene redundancy - the presence of duplicate genes in the genome-and gene pleiotropy, which can lead to several phenotypes based on the same genotype (Morange, 2001).
Biology, when based on reductionism and determinism, seems to lack a systemic perspective that could analyse the interaction of the many factors that influence the behaviour of a system (Van Regenmortel, 2004).

Eo ourth, to better understand complex biological systems and their adaptive behaviour, we need to consider the phenomenon of self-organization, which incorporates many of the ideas mentioned above. Self-organizing systems spontaneously arrange their components and their interactions into adaptive structures with emergent properties. Such structures are able to create new and modify existing strategies to adapt to changing environmental conditions (Coffey, 1998). Complex biological systems, in fact, exist at the 'edge of chaos' where, according to Kauffman (1993), they are able to achieve maximum fitness. Selforganization seems to take place at different levels of biological organization: as a general principle in the organization of the cell itself (Misteli, 2001); in the response of bacterial colonies to chemotactic substrates; and in the brain, which reorganizes itself to learn from experience (Coffey, 1998).

\section{An epistemological rethink is needed to instigate a paradigm shift from the Newtonian model that has dominated science, to an appraisal of complexity that includes both holism and reductionism...}

As mentioned before, adaptation is strictly associated with self-organization. While the latter is an integral feature of the system itself, the former shifts the perspective to the relationship between the system and the environment. Whatever the pressure from the external environment, complex systemsthrough dynamic variations in the degree of interactions between the individual components that form the networks - can adjust and reorganize themselves in a new equilibrium in response to external events. Of course, failures can and do occur, nevertheless, adaptation seems to be the rule rather than the exception (Heylighen et al, 2007).

While arguing about the Darwinian metaphor of the organism adapting to its environment, the American evolutionary biologist Richard Lewontin showed how this idea is based on a dichotomy between inside 
and outside, which disregards the role of the organism in reconstructing its environment (Lewontin, 2000). In fact, organisms neither evolve in isolation nor simply adapt to 'external' factors. On the contrary, they react to and modify their environment according to their own needs. Both organism and environment therefore undergo a continuous process of mutual interaction, which Lewontin termed co-adaptation. A biological system is constrained by its environment, but it also changes its environment. This idea might be applied by analogy, although with different implications, to different levels of organization including, for example, the molecular and the cellular levels (Galtier \& Dutheil, 2007).

7 n this framework, it seems that biology, and somehow science as a whole, needs to reconsider the notion of 'natural laws'. Recent developments in evolutionary biology suggest replacing the idea of a deterministic (prescriptive) law by a non-prescriptive law that just provides a number of constraints. More than actually determining the development of organisms or other complex systems, such laws-if so intended-rather delimit the framework in which they occur. In other words, the 'law as a constraint' establishes the 'rules of the game'. It defines a restricted field of possibilities, within which complex systems develop, but without imposing a unique way of undergoing this process. Other matters should be considered, such as the role of contingencies and of chance, and the way in which the 'players' use these and deal with the existing rules (Ceruti, 1986). It seems that a renewed and more fascinating vision of nature emerges with much more room for variability.

\section{...the reintroduction of the observer has fostered a renewed idea of knowledge}

Of course, many biologists are well aware of the complexity of living systems, but they do not extend this realization to a reappraisal of how to perform science. "When one searches for the 'laws of complexity', one still attaches complexity as a kind of wagon behind the truth locomotive, that which produces laws. A hybrid was formed between the principles of traditional science and the advances towards its hereafter. Actually, one avoids the fundamental problem of complexity, which is epistemological, cognitive, paradigmatic. To some extent, one recognizes complexity, but by decomplexifying it. In this way, the breach is opened, then one tries to clog it: the paradigm of classical science remains, only fissured." (Morin, 2007).

\section{...key notions such as emergence, nonlinearity and self-organization already offer conceptual tools that can contribute to transform and improve science}

An epistemological rethink is needed to instigate a paradigm shift from the Newtonian model that has dominated science, to an appraisal of complexity that includes both holism and reductionism, and which relaxes determinism in favour of recognizing unpredictability as intrinsic to complex systems. Moreover, this 'complex paradigm' requires us to develop 'knowledge of knowledge' and understand its possibilities and limits. Science should not only continue to develop more refined means to analyse its subjects, but also investigate its own approach to generating knowledge (Morin, 1990).

$\mathrm{T}$ his raises the important question of the role of the observer. Classical science has developed by eliminating subjectivity in order to create objective knowledge, but complexity theory has reintroduced the observer as an intrinsic component in the process of gathering knowledge and performing research. The famous uncertainty principle formulated by the German physicist Werner Heisenberg (1901-1976) shows how the 'observer modifies the observation'. This applies not only to quantum physics, but, by extension, can also have practical relevance in the fields of biochemical and biological experimentation. As the Swiss physicist and biologist Eduard Kellenberger (1920-2004) wrote, "for the sub-microscopic world of atoms and molecules, it means that the physical properties of a system-for example, its energy-are uncertain; in particular, these properties depend on the type of observation that is performed on that system, defined by the experimental set-up. In the fashionable field of nano-biotechnology, which examines biological structures at the level of atoms, these relationships are directly relevant. Even at a larger scale, the interactions between an experimental set-up and biological matter give rise to modifications too; for example, bleaching during fluorescence microscopy or the electron-beam-induced alteration-such as burning or carbonization — of a specimen." (Kellenberger, 2004).

Broadly speaking, the reintroduction of the observer has fostered a renewed idea of knowledge. Instead of searching for one fundamental point of observation, scientists need to explore different points of view-which can be vicarious in building a cognitive universe-when describing or explaining a given phenomenon (Ceruti, 1986). Different perspectives can, in fact, complement each other and allow scientists to acquire a more complete knowledge of the phenomenon.

\section{Any constraint can also be an opportunity that, if exploited, allows new possibilities to arise}

One of the most fundamental relationships within this framework is between the system and its environment-the distinction between a point of view that is within the system and one that is external to the system. These two points of view are irreducible and complementary. They emerge from and are defined with respect to one another. The French scientist Henri Atlan, one of the first who tried to define complexity in biology, investigated biological organization from the perspective of information theory. He suggested that, in a hierarchical system, the external observer can be the higher organizational level (Atlan, 1979). In explaining the role of 'noise', he stressed how its 'meaning' depends on the observational perspectives: leading respectively to a decrease or to an increase in information content "whether one is interested in the information transmitted in the channel or in the information transmitted to the observer from a whole system in which the channel is part of a redundant communication network" (Atlan, 1974). For example, a cell would try to suppress noise in its communication pathways as it might lead to unexpected and possibly deleterious effects, but an observer at a higher levelan organ or the organism-would regard noise as a factor that increases variability and therefore overall fitness-provided that the cell is not killed. 
A new theoretical framework should therefore be able to integrate different levels of order while allowing them to maintain their distinctiveness. Such a framework should be grounded on one of the key concepts of complex thinking, unitas multiplex, according to which it is possible to preserve a distinction among what is connected and to connect without reduction (Morin, 1990).

This framework needs to extend to the organization of knowledge. Each domain of knowledge should not be viewed as a closed system-a view that fragments knowledge. Each area should instead participate in a broader project for the development of a science capable of preserving diversity-each field has its own specific features-but also ensuring the integration of the different parts into one common framework.

Biology can therefore be regarded as a discipline with subdisciplines that pertain to different levels of organization (Emmeche et al, 1997). It needs, of course, its own vocabulary and system of conceptualization, and remains partly independent of chemistry and physics. Yet, at the same time, it needs to 'communicate' with them, as well as with other disciplines devoted to the study of systems at higher levels, such as ecology.

A t present, the multifaceted 'complexity paradigm' consists more of a unified way of thinking rather than a finished body of knowledge. Possible applications to the different domains of knowledge need to be further investigated. Nonetheless, key notions such as emergence, nonlinearity and self-organization already offer conceptual tools that can contribute to transform and improve science. Novel approaches and specialized fields of knowledge are already emerging. For example, network biology (Alm \& Arkin, 2003; Barabasi \& Oltvai, 2004) takes advantage of the fact that diverse complex networks, including biological networks, seem to share similar organizational patterns. Some scientists have also stressed how such a change can have beneficial consequences not only at the theoretical level, but also in biomedical research such as drug discovery and vaccine development (Van Regenmortel, 2004).

Furthermore, the epistemological side of complexity offers scientists a chance to reflect on the characteristics and limits of the scientific approach to generating knowledge. Following the notion of the observer, scientists should be more aware that scientific knowledge is always developed within the boundary of given constraints. Yet, this should not be a limiting factor. Any constraint can also be an opportunity that, if exploited, allows new possibilities to arise. Scientists could therefore acquire more complete knowledge of a given phenomenon if they explore it from different cognitive 'horizons', such as points of observation at different hierarchical levels, which by virtue of their vicariousness, cooperate and complement each other.

\section{REFERENCES}

Alm E, Arkin AP (2003) Biological networks. Curr Opin Struct Biol 13: 193-202

Atlan $\mathrm{H}$ (1974) On a formal definition of organization. J Theor Biol 45: 295-304

Atlan H (1979) Entre le Cristal et la Fumée. Paris, France: Éditions du Seuil

Barabasi AL, Oltvai ZN (2004) Network biology: understanding the cell's functional organization. Nat Rev Genet 5: 101-113

Ceruti M (1986) I/ Vincolo e la Possibilità. Milan, Italy: Feltrinelli

Coffey DS (1998) Self-organization, complexity and chaos: the new biology for medicine. Nat Med 4: 882-885

Crick FHC (1966) Of Molecules and Man. Seattle, USA: University of Washington Press

De Haan J (2006) How emergence arises. Ecol Comp/ 3: 293-301

Emmeche C, Køppe S, Stjernfelt F (1997) Explaining emergence: towards an ontology of levels. Journal for General Philosophy of Science 28: 83-119

Galtier N, Dutheil J (2007) Coevolution within and between genes. In Gene and Protein Evolution. Genome Dynamics, JN Volff (ed), vol 3 , pp 1-12. Basel, Switzerland: Karger
Goodall J (1986) The Chimpanzees of Gombe: Patterns of Behaviour. Cambridge, MA, USA: Belknap

Heylighen F, Cilliers P, Gershenson C (2007) Complexity and philosophy. In Complexity, Science and Society, J Bogg, R Geyer (eds). Oxford, UK: Radcliffe (in press)

Horrobin DF (2003) Modern biomedical research: an internally self-consistent universe with little contact with medical reality? Nat Rev Drug Discov 2: 151-154

Kauffman SA (1993) The Origin of Order: Self-Organization and Selection in Evolution. Oxford, UK: Oxford University Press

Kellenberger E (2004) The evolution of molecular biology. EMBO Rep 5: 546-549

Lewontin RC (2000) The Triple Helix. Gene, Organism and Environment. Cambridge, MA, USA: Harvard University Press

Misteli T (2001) The concept of self-organization in cellular architecture. J Cell Biol 155: 181-186

Morange M (2001) The Misunderstood Gene. Cambridge, MA, USA: Harvard University Press Morin E (1990) Introduction á la Pensée Complexe. Paris, France: ESF Éditeur

Morin E (2007) Restricted complexity, general complexity. In Worldviews, Science and Us: Philosophy and Complexity, C Gershenson, D Aerts, B Edmonds (eds), pp 5-29. Singapore: World Scientific

Singer W (2007) Understanding the brain. EMBO Rep 8: S16-S19

Van Regenmortel MHV (2004) Reductionism and complexity in molecular biology. EMBO Rep 5: 1016-1020

Westerhoff HV, Palsson BO (2004) The evolution of molecular biology into systems biology. Nat Biotech 22: 1249-1252

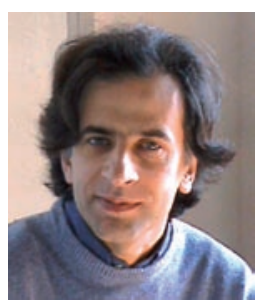

Fulvio Mazzocchi is at the Institute for Atmospheric Pollution of CNR, Monterotondo (Rome), Italy.

E-mail: mazzocchi@iia.cnr.it

doi:10.1038/sj.embor.7401147 